\title{
Forced Axial Flow Between Rotating Concentric Cylinders
}

\section{Citation}

Barcilon, Victor, and Howard C. Berg. 1971. Forced Axial Flow Between Rotating Concentric Cylinders. Journal of Fluid Mechanics 47, no. 03: 469-479.

\section{Published Version}

doi:10.1017/S0022112071001174

\section{Permanent link}

http://nrs.harvard.edu/urn-3:HUL.InstRepos:12220387

\section{Terms of Use}

This article was downloaded from Harvard University's DASH repository, and is made available under the terms and conditions applicable to Other Posted Material, as set forth at http:// nrs.harvard.edu/urn-3:HUL.InstRepos:dash.current.terms-of-use\#LAA

\section{Share Your Story}

The Harvard community has made this article openly available.

Please share how this access benefits you. Submit a story.

\section{Accessibility}




\title{
Forced axial flow between rotating concentric cylinders
}

\author{
By VICTOR BARCILON \\ Department of Mathematics and Institute of Geophysics and Planetary Physics, \\ University of California, Los Angeles
}

AND HOWARD C. BERG†

Department of Biochemistry and Molecular Biology, Harvard University

(Received 5 June 1970 and in revised form 4 January 1971)

Forced axial flow in an annular gap of a cylindrical rotor is investigated analytically and experimentally. At small rotation rates and narrow gap widths, the axial flow is a simple Poiseuille flow over most of the rotor. The distance required for this Poiseuille flow to get established is estimated. An instability is observed at large rotation rates with certain input geometries.

\section{Introduction}

Berg \& Purcell (1967) showed that macro-molecules or small particles can be separated according to effective mass $\ddagger$ when suspended in a rotating annular film of liquid undergoing axial shear flow. The flow is produced by forcing fluid into one end of a cylindrical rotor. If the structure of the shear flow were known, the method could be used to make absolute measurements of effective mass.

In the present paper we investigate the structure of the shear flow analytically and experimentally. We ask whether a Poiseuille flow along the axis can actually be achieved. We find that under certain conditions such a flow is realizable, and that it is possible to determine the distance from the intake in which this flow gets established.

\section{Formulation}

For the purpose of a mathematical analysis of the flow, we can reasonably assume that the fluid is steady, incompressible, homogeneous and viscous. The governing equations of motion are most conveniently written in a rotating frame of reference, viz.

$$
\begin{gathered}
\frac{1}{2} \nabla(\mathbf{q} \cdot \mathbf{q})+(\nabla \times \mathbf{q}) \times \mathbf{q}+2 \mathbf{\Omega} \times \mathbf{q}=-\frac{1}{\rho} \nabla p-\nu \nabla \times(\nabla \times \mathbf{q}), \\
\nabla \cdot \mathbf{q}=0,
\end{gathered}
$$

$\dagger$ Present address: Department of Molecular, Cellular, and Developmental Biology, Boulder, Colorado 80302 .

\# Effective mass is the mass of the particle less the mass of the fluid it displaces. Particles of small effective mass spend a larger fraction of time in rapidly moving regions of fluid than those of large effective mass. Thus, they leave the apparatus sooner. 
where $p, \mathbf{q}$ are the pressure and velocity fields, $\rho$ the constant density, $\nu$ the kinematic viscosity, and $\boldsymbol{\Omega}$ the rotation axis, which coincides with the $z$ axis as well as that of the annular region. We shall denote by $U, V$ and $W$ the velocity components in the radial, zonal and axial directions, respectively.

It is convenient at this stage to introduce dimensionless variables. If $Q$ is the volume rate of fluid injected in the centrifuge, $R$ the mean radius of the annular region and $d$ the half gap width, then we can define a characteristic velocity $W^{*}$ thus:

$$
W^{*}=Q /(4 \pi d R) \text {. }
$$

Denoting by primes dimensionless quantities, we scale the variables as follows:

$$
\left.\begin{array}{c}
r=d r^{\prime}, \quad z=l z^{\prime}, \\
U=(d / l) W^{*} U^{\prime}, \quad V, W=W^{*}\left(V^{\prime}, W^{\prime}\right),
\end{array}\right\}
$$

where

$$
l=d^{3} \Omega / \nu
$$

is taken to be the characteristic length in the $z$ direction. We also have implicitly assumed that $W^{*}$ is the characteristic zonal velocity. We shall see that these assumptions are justified. Finally, in order to scale the pressure, we assume that the main balance in the radial direction is geostrophic, and hence that

This completes the scaling.

$$
P=\left(\rho \Omega W^{*} d\right) P^{\prime}
$$

For the cases of interest, $d \ll R$, so that curvature effects can be neglected, and we can use Cartesian co-ordinates $x$ and $y$ to denote distances along the radial and zonal directions, respectively.

Dropping the primes, and assuming that the flow is axisymmetric, we can rewrite the basic equations (1) and (2) as follows:

$$
\begin{aligned}
\epsilon \mu^{2}\left(U U_{x}+W U_{z}\right)-2 V & =-P_{x}+\mu^{2} U_{x x}+\mu^{4} U_{z z} \\
\epsilon\left(U V_{x}+W V_{z}\right)+2 U & =V_{x x}+\mu^{2} V_{z z} \\
\epsilon\left(U W_{x}+W W_{z}\right) & =-P_{z}+W_{x x}+\mu^{2} W_{z z} \\
U_{x}+W_{z} & =0
\end{aligned}
$$

where

$$
\epsilon=W^{*} / \Omega d
$$

is the Rossby number and

$$
\mu=\nu /\left(\Omega d^{2}\right)
$$

is an Ekman number. The last dimensionless parameter entering into the problem is

$$
\delta=L / l,
$$

where $L$ is the length of the rotor.

The following numerical values can be considered as typical of the centrifuges of interest: $L=30 \mathrm{~cm}, d=10^{-2} \mathrm{~cm}, R=2.5 \mathrm{~cm}, \Omega=10^{3} \mathrm{sec}^{-1}, Q=10^{-2} \mathrm{~cm}^{3}$ sec $^{-1}$. With these numerical values, we see that $\epsilon=3 \times 10^{-3}, \mu=10^{-1}$ and $\delta=3 \times 10^{2}$. We shall therefore confine our attention to the case in which both $\epsilon$ and $\mu$ are small. It should be noted that the smallness of $\mu$ implies that the thickness of the Ekman layer is small compared to the gap of the annulus. This enables us to follow the standard procedure in rotating-fluid flow problems, which 
consists in neglecting the $z$-differentiated terms in the expressions of the viscous force, and in replacing the boundary conditions at $z=0$ and $z=\delta$ by the equivalent Ekman compatibility conditions (Greenspan 1968, p. 92 (2.17.3)). As a result we are led to solve the following boundary-value problem:

$$
\left.\begin{array}{cc}
-2 V=-P_{x}, & 2 U=V_{x x}, \\
0=-P_{z}+W_{x x}, & U_{x}+W_{z}=0
\end{array}\right\} \text { in }|x|<1,0<z<\delta,
$$

with

$$
\begin{aligned}
& U=V=W=0 \quad \text { at } \quad x= \pm 1, \\
& W=-\frac{1}{2} \mu^{\frac{1}{2}} V_{x}+f(x) \text { at } z=0 \\
& W=\frac{1}{2} \mu^{\frac{1}{2}} V_{x}+g(x) \quad \text { at } \quad z=\delta,
\end{aligned}
$$

where $f(x)$ and $g(x)$ are the input and output vertical velocity distributions.

\section{Solution}

Eliminating all fields in favour of $W$, we can rewrite (14) and (15) as follows:

$$
\frac{\partial^{6} W}{\partial x^{6}}+4 \frac{\partial^{2} W}{\partial z^{2}}=0
$$

with

$$
\begin{aligned}
& W=W_{x x x x}=W_{x x x x x}=0 \text { at } \quad x= \pm 1 \text {, } \\
& W_{x x}=\mu^{\frac{1}{2}} W_{z}+f_{x x} \quad \text { at } \quad z=0, \\
& W_{x x}=-\mu^{\frac{1}{2}} W_{z}+g_{x x} \quad \text { at } \quad z=\delta .
\end{aligned}
$$

Using the method of separation of variables, we look for a solution of the form,

$$
W=X(x) Z(z) \text {. }
$$

Substituting (19) in (17), and denoting the separation constant by $\lambda^{6}$, we get

and

$$
\begin{gathered}
Z^{\prime \prime}-\frac{1}{4} \lambda^{6} Z=0, \\
X^{\mathrm{vi}}+\lambda^{6} X=0,
\end{gathered}
$$

where $\lambda$ is real and non-negative (see appendix). The solution of (20) is straightforward:

$$
Z=\left\{\begin{array}{ll}
\text { const. } & \text { if } \lambda=0, \\
e^{-\frac{1}{2} \lambda^{3} z}, & e^{-\frac{1}{2} \lambda^{3}(\delta-z)} \quad \text { if } \quad \lambda \neq 0 .
\end{array}\right\}
$$

The evaluation of $X$ reduces to the solution of the following non-self-adjoint eigenvalue problem:

$$
\left.\begin{array}{c}
X^{\nabla \mathrm{i}}+\lambda^{\mathbf{6}} X=0 \\
X( \pm 1)=X^{\prime \prime \prime}( \pm 1)=X^{\mathrm{v}}( \pm 1)=0 .
\end{array}\right\}
$$

As can be seen from (22), a determination of the eigenvalues $\lambda$ is essential for the computation of the length of the input and output regions.

The solutions of (23) are either even or odd functions and are respectively denoted by $\mathscr{C}_{n}(x)$ and $\mathscr{S}_{n}(x), n=1,2, \ldots$, where

$\mathscr{C}_{n}(x)=\cos \alpha_{n} x-\frac{\sin \frac{3}{2} \alpha_{n}}{\sinh \frac{\sqrt{3} 3}{2} \alpha_{n}} \sin \frac{1}{2} \alpha_{n} x \sinh \frac{\sqrt{3}}{2} \alpha_{n} x-\frac{\cos \frac{3}{2} \alpha_{n}}{\cosh \frac{\sqrt{3}}{2} \alpha_{n}} \cos \frac{1}{2} \alpha_{n} x \cosh \frac{\sqrt{ } 3}{2} \alpha_{n} x$, 
and

$$
\begin{aligned}
\mathscr{S}_{n}(x)=\sin \beta_{n} x-\frac{\sin \frac{3}{2} \beta_{n}}{\sinh \frac{\sqrt{3}}{2} \beta_{n}} \cos \frac{1}{2} \beta_{n} x \sinh \frac{\sqrt{3}}{2} \beta_{n} x & \\
& +\frac{\cos \frac{3}{2} \beta_{n}}{\cosh \frac{\sqrt{3}}{2} \beta_{n}} \sin \frac{1}{2} \beta_{n} x \cosh \frac{\sqrt{3}}{2} \beta_{n} x,
\end{aligned}
$$

where the eigenvalues are the roots $\alpha_{n}$ and $\beta_{n}$ of the following transcendental equations:

$$
\cos \frac{3}{2} \alpha_{n} \cos \frac{1}{2} \alpha_{n} \tanh \frac{\sqrt{3}}{2} \alpha_{n}+\sin \frac{3}{2} \alpha_{n} \sin \frac{1}{2} \alpha_{n} \operatorname{coth} \frac{\sqrt{3}}{2} \alpha_{n}=\sqrt{ } 3 \sin \alpha_{n},
$$

and

$$
\cos \frac{3}{2} \beta_{n} \sin \frac{1}{2} \beta_{n} \tanh \frac{\sqrt{3}}{2} \beta_{n}-\sin \frac{3}{2} \beta_{n} \cos \frac{1}{2} \beta_{n} \operatorname{coth} \frac{\sqrt{3}}{2} \beta_{n}=\sqrt{ } 3 \cos \beta_{n} .
$$

In addition, for $\lambda=0$, we have

$$
\mathscr{C}_{0}(x)=1-x^{2}
$$

The calculation of the $\alpha_{n}$ and $\beta_{n}$ could be done numerically, but, as we shall presently see, this is not necessary. If we assume, subject to a posteriori verification, that the $\alpha_{n}$ and $\beta_{n}$ are large, we can replace the hyperbolic functions by their asymptotic values. We thus obtain

$$
\left.\begin{array}{l}
\alpha_{n} \simeq \frac{1}{6} \pi+(n-1) \pi, \\
\beta_{n} \simeq \frac{2}{3} \pi+(n-1) \pi,
\end{array}\right\} \quad n=1,2, \ldots
$$

Except for $\alpha_{1}$, all the other $\alpha_{n}$ and $\beta_{n}$ are indeed large. To determine $\alpha_{1}$ more accurately, numerical calculations were performed. These calculations revealed that there are no roots in the neighbourhood of $\frac{1}{6} \pi$, and that the first zero of (26) is $\alpha_{1}=\frac{7}{6} \pi !$ In other words, (29) should read

$$
\left.\begin{array}{l}
\alpha_{n} \simeq \frac{7}{6} \pi+(n-1) \pi, \\
\beta_{n} \simeq \frac{2}{3} \pi+(n-1) \pi,
\end{array}\right\} \quad n=1,2, \ldots .
$$

This result is rather fortunate from the view-point of the centrifuge design.

The complete solution of the problem is:

$$
\begin{aligned}
W=A\left(1-x^{2}\right)+\sum_{n=1}^{\infty}[ & \left.a_{n} \sinh \frac{1}{2} \alpha_{n}^{3}(\delta-z)+b_{n} \sinh \frac{1}{2} \alpha_{n}^{3} z\right] \mathscr{C}_{n}(x) \\
& +\sum_{n=1}^{\infty}\left[a_{n}^{\prime} \sinh \frac{1}{2} \beta_{n}^{3}(\delta-z)+b_{n}^{\prime} \sinh \frac{1}{2} \beta_{n}^{3} z\right] \mathscr{S}_{n}(x) .
\end{aligned}
$$

The coefficients $A, a_{n}, b_{n}, a_{n}^{\prime}$ and $b_{n}^{\prime}$ are determined from the actual input and output conditions. Their evaluation can be greatly simplified if the terms of order $\mu$ in the boundary conditions (15) are neglected, i.e. if the Ekman layer suction is neglected. All that is now required is the construction of a complete set of functions orthogonal to $\left\{1-x^{2}, \mathscr{C}_{n}(x), \mathscr{S}_{n}(x)\right\}$; to that effect we consider the adjoint of (23), viz.

$$
\left.\begin{array}{c}
Y^{\mathrm{vi}}+\lambda^{6} Y=0, \\
Y^{\prime}( \pm 1)=Y^{\prime \prime \prime}( \pm 1)=Y^{\mathrm{iv}}( \pm 1)=0 .
\end{array}\right\}
$$

The solutions of this eigenvalue problem, again separated into even and odd functions denoted by ' $C_{n}(x)$ ' and ' $S_{n}(x)$ ' are:

$$
\begin{aligned}
C_{n}(x)=\cos \alpha_{n} x & +\left(\frac{\sqrt{ } 3 \sin \frac{3}{2} \alpha_{n}}{2 \sinh \frac{\sqrt{3}}{2} \alpha_{n}}+\frac{\cos \frac{3}{2} \alpha_{n}}{2 \cosh \frac{\sqrt{3}}{2} \alpha_{n}}\right) \cos \frac{1}{2} \alpha_{n} x \cosh \frac{\sqrt{3}}{2} \alpha_{n} x \\
& +\left(\frac{\sin \frac{3}{2} \alpha_{n}}{2 \sinh \frac{\sqrt{3}}{2} \alpha_{n}}-\frac{\sqrt{ } 3 \cos \frac{3}{2} \alpha_{n}}{2 \cosh \frac{\sqrt{3}}{2} \alpha_{n}}\right) \sin \frac{1}{2} \alpha_{n} x \sinh \frac{\sqrt{3}}{2} \alpha_{n} x,
\end{aligned}
$$




$$
\begin{aligned}
S_{n}(x)=\sin \beta_{n} x- & \left(\frac{\sqrt{ } 3 \sin \frac{3}{2} \beta_{n}}{2 \sinh \frac{\sqrt{3}}{2} \beta_{n}}+\frac{\cos \frac{3}{2} \beta_{n}}{2 \cosh \frac{\sqrt{3}}{2} \beta_{n}}\right) \sin \frac{1}{2} \beta_{n} x \cosh \frac{\sqrt{3}}{2} \beta_{n} x \\
& +\left(\frac{\sin \frac{3}{2} \beta_{n}}{2 \sinh \frac{\sqrt{3}}{2} \beta_{n}}-\frac{\sqrt{ } 3 \cos \frac{3}{2} \beta_{n}}{2 \cosh \frac{\sqrt{3}}{2} \beta_{n}}\right) \cos \frac{1}{2} \beta_{n} x \sinh \frac{\sqrt{3}}{2} \beta_{n} x,
\end{aligned}
$$

where $n=1,2, \ldots$ and $\alpha_{n}$ and $\beta_{n}$ are the roots of (26) and (27). In addition, corresponding to the eigenvalue $\lambda=0$, we also have

$$
C_{0}(x)=1 \text {. }
$$

Since $\left\{1-x^{2}, \mathscr{C}_{n}(x), \mathscr{S}_{n}(x)\right\}$ and $\left\{1, C_{n}(x), S_{n}(x)\right\}$ form bi-orthogonal, complete sets of functions (see appendix), we deduce that

$$
\begin{aligned}
a_{n}= & \frac{\int_{-1}^{+1} f(x) C_{n}(x) d x}{\int_{-1}^{+1} \mathscr{C}_{n}(x) C_{n}(x) d x} \frac{1}{\sinh \frac{1}{2} \alpha_{n}^{3} \delta} \\
b_{n}= & \frac{\int_{-1}^{+1} g(x) C_{n}(x) d x}{\int_{-1}^{+1} \mathscr{C}_{n}(x) C_{n}(x) d x} \frac{1}{\sinh \frac{1}{2} \alpha_{n}^{3} \delta}
\end{aligned}
$$

with similar expressions for $a_{n}^{\prime}$ and $b_{n}^{\prime}$. For the case $l \ll L$ (i.e. $\delta \gg 1$ ), the coefficients $a_{n}, b_{n}, a_{n}^{\prime}$ and $b_{n}^{\prime}$ are exponentially small. Hence, except in the immediate vicinity of the input and output regions, the axial velocity $W$ is equal to $A\left(1-x^{2}\right)$. It is a simple matter to check that over this intermediary region the other velocity components are zero. As a result, outside of the input and output regions, the flow is a simple Poiseuille flow. This result holds regardless of the detailed structure of the prescribed velocity distribution $f(x)$ and $g(x)$. Furthermore, the flows in the input and output regions are uncoupled. The maximum extent of these regions is $2 \beta_{1}^{-3}$, or in dimensional units

$$
h=\frac{27}{4 \pi^{3}} \frac{\Omega d^{3}}{\nu} \text {. }
$$

However, if the injection velocity is symmetric, this length is reduced by a factor of five. Indeed, if $f(x)=f(-x)$, then all the $a_{n}^{\prime}$ vanish and the Poiseuille flow is established after a distance of

$$
\left(432 / 342 \pi^{3}\right)\left(\Omega d^{3} / \nu\right) .
$$

The axial mass transport is accomplished entirely by the Poiseuille flow component. This can be seen from the fact that 1 is orthogonal to $\mathscr{C}_{n}(x)$ and $\mathscr{S}_{n}(x)$. As a result, we can evaluate the coefficient $A$ by simply averaging (31), viz.

$$
\int_{-1}^{+1} W d x=A \int_{-1}^{+1}\left(1-x^{2}\right) d x
$$

and recalling that in our non-dimensionalization the average axial flux equals 2 . Hence,

$$
A=\frac{3}{2} \text {. }
$$




\section{Experiments}

The geometry chosen was that thought useful in a practical centrifuge (figure 1). The rotor was spun horizontally in a variable-speed lathe. The tube from the chuck end of the rotor extended through the headstock to a small

(i) $7 \cdot 10 \mathrm{~g} \mathrm{Na}_{2} \mathrm{HPO}_{4}$ in $500 \mathrm{ml}$. water

(ii) $6.90 \mathrm{~g} \mathrm{NaH}_{2} \mathrm{PO}_{4} \cdot \mathrm{H}_{2} \mathrm{O}$ in $500 \mathrm{ml}$. water, plus enough $\mathrm{NaCl}$ to match the density to that of (i) (about $3 \mathrm{~g}$ ) $\dagger$

(iii) $0.20 \mathrm{~g}$ bromothymol blue (Fisher) in $32 \mathrm{ml} .0 .01 \mathrm{~m}-\mathrm{NaOH}$ plus $468 \mathrm{ml}$. water (final $\mathrm{pH}=6 \cdot 8$ )

Yellow solution: $100 \mathrm{ml}$. (ii), $250 \mathrm{ml}$. (iii), water to $21 . \$$

Blue solution: $100 \mathrm{ml}$. (i), $250 \mathrm{ml}$. (iii), water to $21 . \pm$

i (i) and (ii) were weighed in the same $50 \mathrm{ml}$. volumetric flask to an accuracy of about $1 \mathrm{mg}$, and $\mathrm{NaCl}$ was added to (ii) until the weights were indistinguishable.

† The same volumetric flasks were used for preparing both of these solutions.

TABLe 1. Scheme used for preparing yellow and blue solutions of matched density. The water was distilled
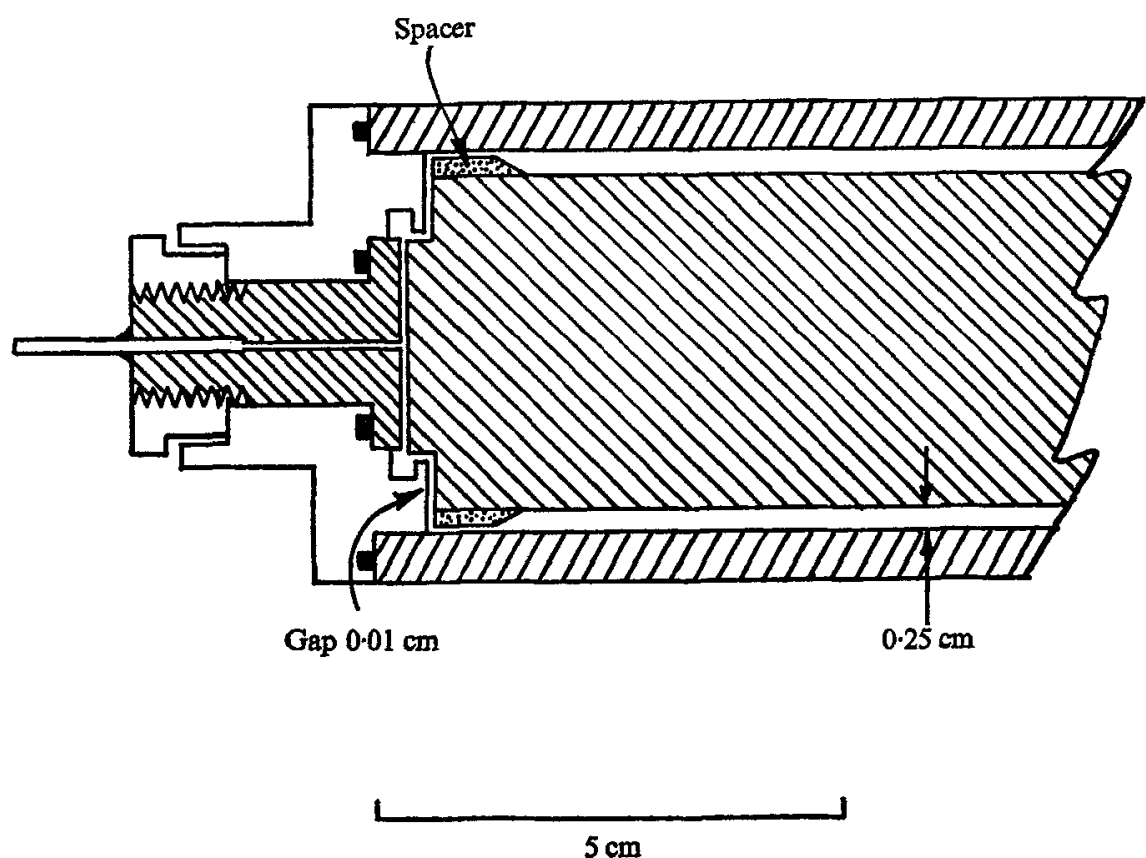

FIGURE 1. A schematic cross-sectional view of the horizontal rotor. The left end is shown; the right was identical. A plexiglass tube and cylindrical core (shaded) were held together by stainless-steel caps and nuts (clear) and sealed with viton-A O-rings (black). Fluid was pumped in or out of the rotor through stainless tubing, an axial hole, four radial holes (two are shown) and a series of $0.01 \mathrm{~cm}$ gaps (not to scale). Spacers of plexiglas (stippled) at either end of the annular gap could be removed (for injection radially at the smallest radius of the gap, rather than axially at the largest radius). The rotor was machined to a tolerance of $0.001 \mathrm{in}$. and chucked so that the run-out was less than 0.001 in. Other dimensions are given in table 2 . 
teflon-gasketed rotary joint; the one at the other end led to a similar joint mounted in the tailstock. The flow profile (or, more precisely, its integral over the width of the gap) was visualized by using solutions of bromothymol blue (an acid-base indicator, $\mathrm{pI}=6 \cdot 8$ ). Solutions of suitable colour stability and intensity were prepared by mixing the indicator with sodium phosphate buffers (buffer $\mathrm{pK}=6 \cdot 8=\mathrm{pI}$ ), table 1 . The rotor was filled with the yellow solution and then $20 \mathrm{ml}$. was displaced with the blue. The final distribution of colour was recorded photographically on $35 \mathrm{~mm}$ Kodachrome II by back-lighting the rotor with an electronic flash from behind a sheet of tracing paper. $\dagger$ Colour standards were

$\begin{array}{cccc}\Omega\left(\mathrm{rad} \mathrm{sec}^{-1}\right) & h=27 \Omega d^{3} / 4 \pi^{3} \nu & \epsilon=\frac{W^{*}}{\Omega d} & \mu=\frac{\nu}{\Omega d^{2}} \\ \Omega_{1}=58 & 2.56 & 1.5 \times 10^{-2} & 1.1 \times 10^{-2} \\ \Omega_{2}=143 & 6.32 & 6.1 \times 10^{-3} & 4 \cdot 3 \times 10^{-3} \\ \Omega_{3}=229 & 9.12 & 3.8 \times 10^{-3} & 2.7 \times 10^{-3}\end{array}$

$\dagger d=0.127 \mathrm{~cm}, L=17.8 \mathrm{~cm}$ (spacers removed), $R=1.91 \mathrm{~cm}, v=10^{-2} \mathrm{~cm}^{2} \mathrm{sec}^{-1}$, $Q=0.33 \mathrm{~cm}^{3} \mathrm{sec}^{-1}$, and $W^{*}=0.11 \mathrm{~cm} \mathrm{sec}^{-1}$.

Table 2. Parameters pertaining to the experiments of figures 1 and 2. $\dagger$

recorded in the same way after the rotor had been filled with known mixtures of the two solutions. A composite slide of the standards was projected alongside slides of the different experiments. The latter were viewed through a mask and the positions along the rotor at which the colours matched were noted.

A number of results are shown in figure 2. Parameters pertaining to these experiments are given in table 2. The dimensions of the rotor, the volume of the plumbing and the volume of the injected solution were known, so it was possible to calculate what the colour distribution would have been were the flow strictly parabolic. This distribution is shown by the dashed curves in figure 2 , denoted $P$. A distribution of this shape was observed when the rotor was stationary, but the colour front was not as axially symmetric as when the rotor was spinning. At $\Omega_{1}$, the colour distributions were essentially the same for a given input geometry regardless of the output geometry (compare in figure $2,(a)$ to $(b)$ and (c) to $(d)$ ); therefore, the flows at the ends of the rotor were not coupled. The distributions at $\Omega_{1}$ were displaced downstream from $P$ by about $1 \mathrm{~cm}$. Both results are consistent with the theory which predicts a maximum $e$-folding length $h=2.56 \mathrm{~cm}$, table 2 . At $\Omega_{2}$ and $\Omega_{3}$, the flows at the ends of the rotor were coupled since the colour distributions depended on the output geometry (compare $(a)$ to $(b)$ and $(c)$ to $(d)$ ). Departures from $P$ were severe in configurations $(a)$ and $(d)$. These results are also consistent with the theory which predicts no intermediary Poiseuille flow region for small values of $\delta$.

Several observations suggest that inertial effects were not entirely absent. For instance, when the flow rates were reduced by a factor of 4 , the dips in the distribution curves near the input became somewhat more shallow and the

$\dagger$ The flash was fast enough to freeze all motion. 

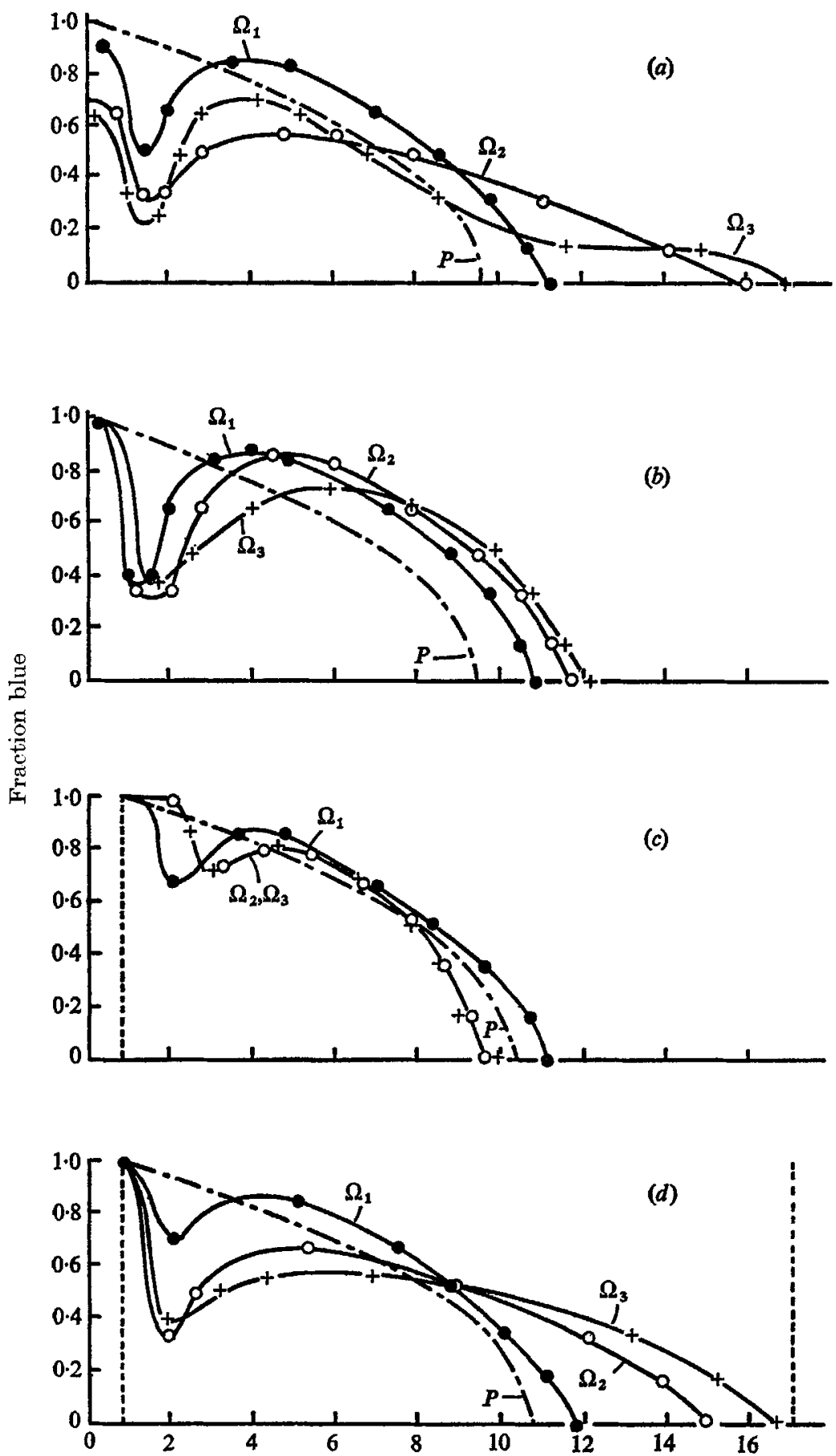

Distance from input $(\mathrm{cm})$

Figure 2. Curves showing the colour distributions expected for a parabolic profile $(P)$ and those observed at rotation $\Omega_{1}, \Omega_{2}$ and $\Omega_{3}$ when $(a)$ input and output were at the smallest radius of the gap; $(b)$ input was at the smallest radius, output at the largest radius; (c) input was at the largest radius, output at the smallest radius; and $(d)$ input and output were at the largest radius of the gap. The vertical dotted lines indicate the effective extent of the spacers. See table 2. 
curves shifted towards $P$ (see figure 2). When the flow rates were increased by a factor of 4 , the dips deepened and the curves shifted away from $P$. These changes were more striking in configuration $(b)$ than in $(d)$. It is worth noting in this connexion that, whereas the linear equations (14) admit solutions for which $U$ and $V$ are even functions of $x$ and $W$ is an odd function of $x$, the 'inertial' equations,

$$
\left.\begin{array}{rl}
-2 V & =-P_{x} \\
\epsilon\left(U V_{x}+W V_{z}\right)+2 U & =V_{x x} \\
\epsilon\left(U W_{x}+W V_{z}\right) & =-P_{z}+W_{x x} \\
U_{x}+W_{z} & =0
\end{array}\right\}
$$

do not allow solutions which have these symmetries. The intake configuration is also likely to be responsible for an even more important inertial effect, namely flow instability. Indeed, at $\Omega_{2}$ and $\Omega_{3}$, for all flow rates tested $\left(0 \cdot 06-2 \cdot 0 \mathrm{~cm}^{3} \mathrm{sec}^{-1}\right)$, long axial streaks were observed (figure 3 , plate 1 ) for configurations $(a)$ and $(b)$ but not for configurations $(c)$ and $(d) . \dagger$ No such zonal variations were seen at $\Omega_{1}$ for flow rates up to $2 \cdot 5 \mathrm{~cm}^{3} \mathrm{sec}^{-1}$. One can speculate that the instability, which must be rooted in the intake region, is a shear instability connected with the velocity component in the zonal direction.

The bromothymol blue technique displays the integral of the shear profile, not the profile itself. We tried to study the latter by mounting a much larger set of concentric cylinders on the vertical axis of a rotating table. The moulded plexiglass cylinders used were not precisely round. The gap was illuminated momentarily from the side with a $2 \mathrm{~cm}$-wide strip of light (fixed to the table) and photographed from the front through a water-filled rectangular box cemented to the rotor (to reduce diffraction). The bottom of the gap was filled with $0 \cdot 4 \mathrm{~cm}$ diameter glass beads to a depth of about $3 \mathrm{~cm}$, and water was injected continuously through the beads from below. The shear profile was visualized by the pulse-addition of a few $\mathrm{ml}$ of a suspension of aluminium powder. Photographs of one such pulse are shown in figure 4 (plate 2$): d=0 \cdot 64 \mathrm{~cm}, L=132 \mathrm{~cm}, R=13 \cdot 3$ $\mathrm{cm}, \nu=10^{-2} \mathrm{~cm} \mathrm{sec}^{-1}, Q=7 \cdot 3 \mathrm{~cm}^{3} \mathrm{sec}^{-1}, W^{*}=0.068 \mathrm{~cm} \mathrm{sec}-1, \Omega=3 \cdot 1 \mathrm{rad} \mathrm{sec}^{-1}$, $432 \Omega d^{3} / 343 \pi^{3} \nu=3.2 \mathrm{~cm}, \epsilon=3.4 \times 10^{-2}$, and $\mu=7.9 \times 10^{-3}$. As far as one can tell, the flow became parabolic within a distance of about $5 \mathrm{~cm}$. At lower flow rates the pulse patterns were badly skewed. The powder drifted around even when the circulating pump was turned off, and the same thing happened with polystyrene particles of specific gravity 1.02 ; these effects were probably due to convection.

Zonal variations were studied by mixing a relatively large amount of aluminium powder with the fluid in the rotor and by using oblique illumination. Longitudinal streaks were present in photographs of the bottom $25 \mathrm{~cm}$ of the rotor at a flow rate of $7.3 \mathrm{~cm}^{3} \mathrm{sec}^{-1}$ at angular velocities of 4.5 and $6 \mathrm{rad} \mathrm{sec}^{-1}$; they were less pronounced at $3 \mathrm{rad} \mathrm{sec}^{-1}$ and absent at $1.5 \mathrm{rad} \mathrm{sec}^{-1}$. The distance between the streaks was, again, about equal to the width of the gap. The streaks were not seen at a flow rate of $0.3 \mathrm{~cm}^{3} \mathrm{sec}^{-1}$.

$\dagger$ In matching colours to obtain the curves of figure 2, the streaks were ignored, and a rough estimate was made of the average local colour. 
We are grateful to E.M. Purcell for his help during the early phases of this work, and to J.D. Baker for the use of his rotating table. The work was supported by Ivan Sorvall, Inc., Newton, Connecticut 06470.

\section{Appendix. Properties of the eigensolutions $\left\{\lambda_{n}, X_{n}(x)\right\}$}

In order to deduce some of the properties of $\left\{\lambda_{n}, X_{n}(x)\right\}$, we shall consider two closely related eigenvalue problems, namely, the adjoint problem (34) for $\left\{\lambda_{n}, Y_{n}(x)\right\}$ and the following self-adjoint eigenvalue problem:

$$
\left.\begin{array}{l}
\frac{d^{6} Z}{d x^{6}}+\lambda^{6} Z=0, \\
\frac{d^{2} Z}{d x^{2}}=\frac{d^{4} Z}{d x^{4}}=\frac{d^{5} Z}{d x^{5}}=0 \quad \text { at } \quad x= \pm 1
\end{array}\right\}
$$

It is important to note that

$$
Z(x)=\frac{d X}{d x} \quad \text { and } \quad Y(x)=\frac{d^{2} X}{d x^{2}} .
$$

TheOREM. $\lambda$ is real and non-negative.

Denoting complex conjugation by an over-bar, we can write

$$
\frac{d^{6} \bar{X}_{n}}{d x^{6}}+\bar{\lambda}_{n}^{6} \bar{X}_{n}=0, \quad \frac{d^{6} Y_{m}}{d x^{6}}+\lambda_{m}^{6} Y_{m}=\mathbf{0} .
$$

Multiplying the first equation by $Y_{m}$, the second by $\bar{X}_{n}$, subtracting the resulting equations, and integrating over $(-1,1)$, we get

$$
\int_{-1}^{+1}\left\{Y_{m} \frac{d^{6} \bar{X}_{n}}{d x^{6}}-\bar{X}_{n} \frac{d^{6} Y_{m}}{d x^{6}}\right\} d x+\left(\overline{\lambda_{n}^{6}}-\lambda_{m}^{6}\right) \int_{-1}^{+1} \bar{X}_{n} Y_{m} d x=0
$$

Since $Y$ is a solution of the adjoint problem, the first integral in (A 4) vanishes, and so

$$
\left.\overline{\left(\lambda_{n}^{6}\right.}-\lambda_{m}^{6}\right) \int_{-1}^{+1} \bar{X}_{n} Y_{m} d x=0 .
$$

In particular, for $n=m$ the above becomes

$$
\left.\overline{\left(\lambda_{n}^{6}\right.}-\lambda_{n}^{6}\right) \int_{-1}^{+1} \bar{X}_{n} \frac{d^{2} X_{n}}{d x^{2}} d x=0
$$

after $Y_{n}$ has been replaced by its expression in terms of $X_{n}$. Integrating by parts once again, and using the boundary conditions on $X_{n}$, we deduce that

$$
\left(\overline{\lambda_{n}^{6}}-\lambda_{n}^{6}\right) \int_{-1}^{+1}\left|\frac{d X_{n}}{d x}\right|^{2} d x=0
$$

Therefore $\lambda_{n}^{6}$ is real. Note that, if $\lambda_{n}^{\mathbf{6}} \neq \lambda_{m}^{6}$, (A 5) implies that

$$
\int_{-1}^{+1} \bar{X}_{n} Y_{m} d x=0 \quad \text { for } \quad \lambda_{n}^{6} \neq \lambda_{m}^{6}
$$

i.e. that $\left\{X_{n}(x)\right\}$ and $\left\{Y_{n}(x)\right\}$ are bi-orthogonal sequences.

In order to prove that $\lambda^{6}$ is non-negative, we simply multiply the equation for $X$ by $d^{2} \bar{X} / d x^{2}$, and integrating it over $(-1,1)$ we obtain

$$
\left[\frac{d^{2} \bar{X}}{d x^{2}} \frac{d^{5} X}{d x^{5}}-\frac{d^{3} \bar{X}}{d x^{3}} \frac{d^{4} X}{d x^{4}}\right]_{-1}^{+1}+\int_{-1}^{+1}\left|\frac{d^{3} X}{d x^{3}}\right|^{2} d x+\lambda^{6}\left[X \frac{d \bar{X}}{d x}\right]_{-1}^{+1}-\lambda^{6} \int_{-1}^{+1}\left|\frac{d X}{d x}\right|^{2} d x=0
$$


On account of the boundary conditions, this equation reduces to

$$
\int_{-1}^{+1}\left|\frac{d^{3} X}{d x^{3}}\right|^{2} d x=\lambda^{6} \int_{-1}^{+1}\left|\frac{d X}{d x}\right|^{2} d x
$$

Without loss of generality, we can therefore consider $\lambda$ itself to be real and non-negative.

Note that, if $\left(\lambda_{n}, X_{n}\right)$ is an eigensolution, so is $\left(\lambda_{n}, \bar{X}_{n}\right)$; it is therefore always possible to construct real eigenfunctions associated with $\lambda_{n}$. Without loss of generality, we shall therefore assume that $X_{n}$ is real.

THEOREM 2. $\left\{X_{n}(x)\right\}$ constitutes a complete set of functions over $(-1,1)$.

Let us assume that $\left\{X_{n}(x)\right\}$ is not a complete set. Then there exists an $L_{2}(-1,1)$ function, say $\psi(x)$, such that

$$
\int_{-1}^{+1} \psi(x) X_{n}(x) d x=0 \text { for } n=0,1,2, \ldots
$$

and

$$
\int_{-1}^{+1}[\psi(x)]^{2} d x \neq 0
$$

If we define $\phi(x)$ as follows:

$$
\phi(x)=\int_{-1}^{x} \psi(\xi) d \xi
$$

then we can rewrite (A 10) thus:

$$
\int_{-1}^{+1} \frac{d \phi}{d x} X_{n}(x) d x=0 \text { for } \quad n=0,1,2, \ldots
$$

Integrating by parts and using the boundary conditions for $X_{n}$ as well as (A 2) we deduce that

$$
\int_{-1}^{+1} \phi(x) Z_{n}(x) d x=0 \text { for } n=0,1,2, \ldots
$$

Furthermore, it is clear, from (A 11) and (A 12), that

$$
\int_{-1}^{1}[\phi(x)]^{2} d x \neq 0
$$

But (A 14) and (A 15) imply that $\left\{Z_{n}(x)\right\}$ is not a complete set of functions in $L_{2}(-1,1)$, which is a contradiction, in view of the fact that $\left\{Z_{n}(x)\right\}$ arises from a self-adjoint eigenvalue problem (see Coddington \& Levinson 1955, p. 57). Therefore, $\left\{X_{n}(x)\right\}$ is a complete set of functions in $L_{2}(-1,1)$.

\section{REFERENCES}

Berg, H. C. \& Purcelt, E. M. 1967 Proc. Natn. Acad. Sci. (U.S.A.) 58, 1821.

Coddington, E. A. \& Levinson, N. 1955 The Theory of Ordinary Differential Equations. McGraw-Hill.

Gremaspan, H. P. 1968 The Theory of Rotating Fluids. Cambridge University Press. 


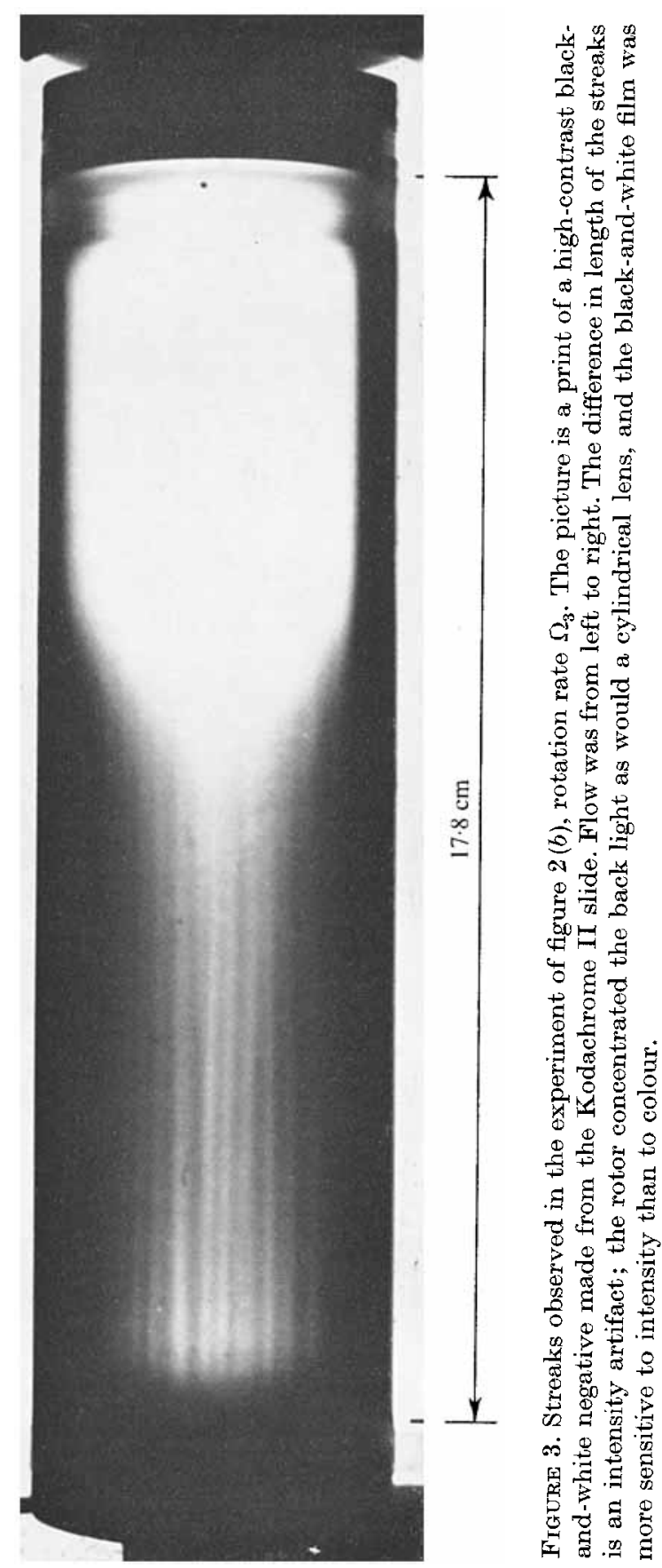




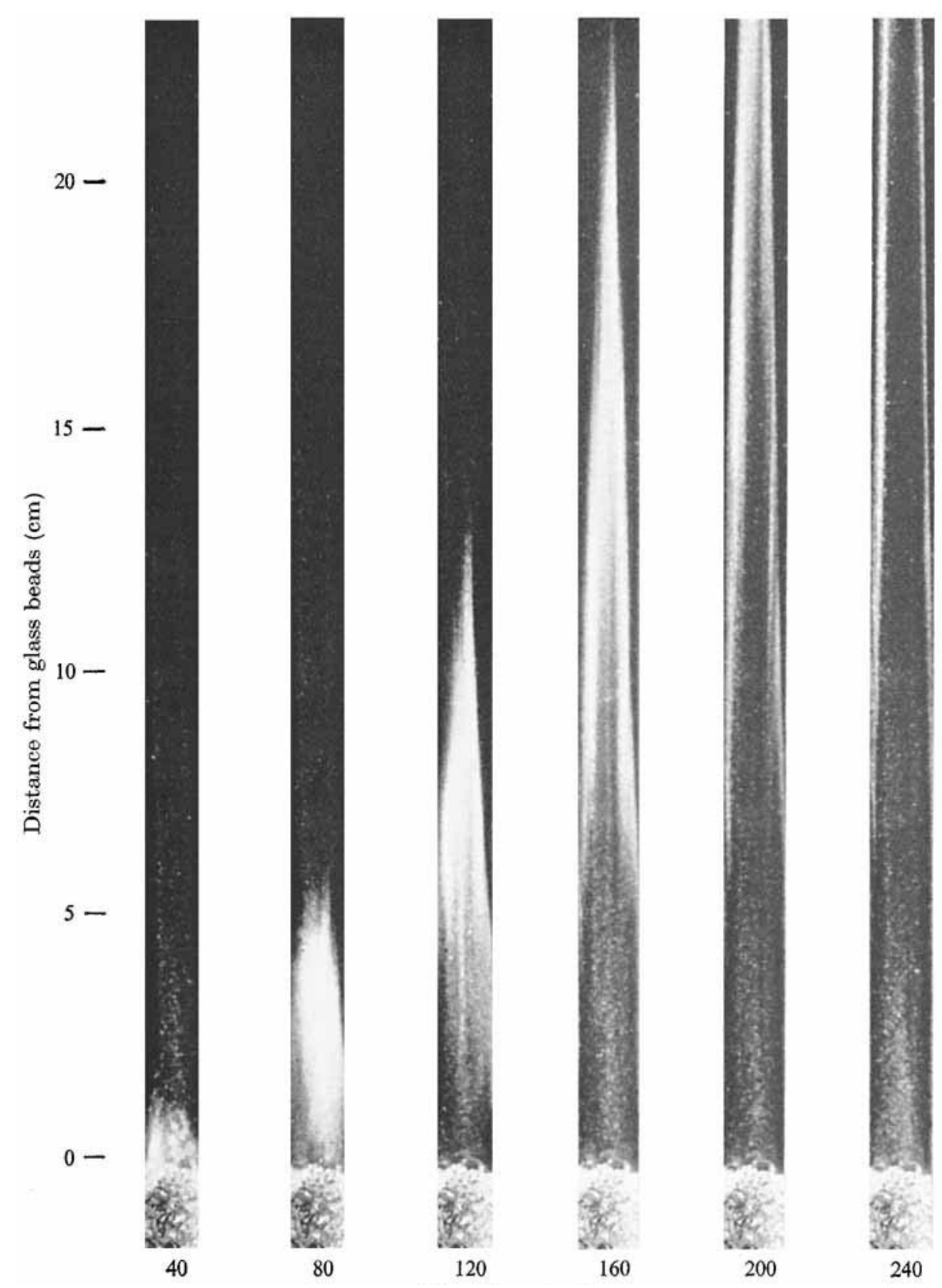

Figure 4. A sequence of photographs of the bottom $25 \mathrm{~cm}$ of the gap of the vertical rotor after injection of aluminum powder. 